\title{
Biomarker mRNAs as prognostic tools for lymph node analysis in colorectal cancer
}

\author{
Sten Hammarström*,1 \\ ${ }^{1}$ Department of Clinical Microbiology, Infection \& Immunology, Umeå University, SE-90185 Umeå, Sweden \\ *Author for correspondence: sten.hammarstrom@umu.se

\section{“there is an urgent need for complementary methods to histopathology"}

First draft submitted: 8 May 2019; Accepted for publication: 14 May 2019; Published online: 26 June 2019

Keywords: lymphnode analysis $\bullet$ mRNA expression levels $\bullet$ selection of multiple biomarkers $\bullet$ tumor aggressiveness - tumor cell load

Prognostic indicators are of utmost importance for physician in the management of disease in cancer patients - in particular for patients suffering from cancers where there is a significant risk that the primary treatment does not result in complete cure. Colocrectal cancer (CRC) is the second most deadly cancer worldwide. In approximately $50 \%$ of CRC patients with tumor-cell-positive lymph nodes (i.e., Stage III CRC [anyTN1-2M0]), and about $25 \%$ of CRC patients with no detected tumor-cell positive lymph nodes ( i.e., Stage I [T1-2 N0M0] and Stage II [T3-4N0M0]), disease will recur after curative surgery [1,2]. For CRC, the single most important prognostic indicator is the presence - or absence - of tumor cell metastasis in regional lymph nodes in the resected specimen [3]. Presence of lymph node metastasis is generally determined by histopathological examination of hematoxylin and eosin (H\&E) stained tissue sections of resected regional lymph nodes.

Present guidelines require that at least 12 lymph nodes should be examined [4,5]. However, the results obtained by H\&E staining method, which forms the base for predicting prognosis in CRC, has problems resulting in both undertreatment of Stage I and II patients and overtreatment of Stage III patients. Major problems are that only a small volume of the lymph node can be analyzed in clinical practice (generally less than 1\%), furthermore that the lymphatic field surrounding the primary tumor generally is large containing up to 50-100 lymph nodes, each of which potentially harboring tumor cells. Thus, complete examination of the entire lymphatic field is a formidable task. Furthermore, identification of tumor cells in stained tissue sections is difficult - requiring an experienced pathologist. Finally, morphology alone gives no information about the aggressiveness of the tumor cells. In other words, whether the tumor cells are aggressive with capacity to leave the lymph node and ultimately form metastases at distant sites (most commonly in the liver, lungs and peritoneum) which may be fatal.

In my view, there is an urgent need for complementary methods to histopathology. One avenue for improvement, which has been used to a limited degree in CRC, is immunopathology using specific antibodies for discriminating molecules characterizing the tumor cells. However, limiting factors are still the volume challenge and the labor intensity of immunohistopathological methods.

Molecular methods have the potential to overcome most of the problems associated with histo- and immunopathology. Analysis of biomarker expression at the mRNA level has several advantages, importantly among which are that the expression levels of several biomarkers can easily be analyzed in the same RNA extract and that a much larger volume of the lymph node is available for analysis. Practically about $50 \%$ of the volume, if the node is divided into two halves - one half for RNA extraction, the other half for histopathology and/or immunopathology. Real-time quantitative reverse transcriptase-PCR (qRT-PCR) analysis is the most useful method for mRNA analysis of biomarkers. It has been used successfully with biomarkers such as CEACAM5, KLK6, CK20 and GUCY2C [6-9,11]. It should be noted that there are alternative methods for determination of biomarker mRNAs, notably next-generation sequencing, among them RNA-seq but at present qRT-PCR is the method of choice for use in clinical practice. When determining biomarker mRNA expression, it is important to also determine the expression level of a house-keeping gene, ideally a gene that does not vary to much between cell types and cell 
cycle stages. Our experience is that $18 \mathrm{~S}$ rRNA is a most useful house-keeping gene in the analysis of tumor cells in lymph nodes by qRT-PCR [10]. A second point is that biomarker mRNA levels should be expressed as copies of mRNA rather than relative quantities as $\Delta C_{\mathrm{t}}$ and fold differences based on $\Delta \Delta C_{\mathrm{t}}$ values. Thus, a copy standard is preferably prepared for each biomarker under investigation.

Factors to be considered in biomarker selection and assay construction in order to obtain prognostic information from tumor mRNA analysis of lymph nodes in CRC are:

- Biomarker mRNAs for tumor cell detection should not be expressed in immune cells as prerequisite for use [6];

- At least one highly expressed biomarker mRNA with low variation between tumors must be included. Can serve as a measure of tumor cell number as is the case for CEACAM5 mRNA [6,11];

- It is valuable to include biomarker mRNAs related to aggressiveness, for example, expressed ectopically in tumor cells as is the case for $K L K 6$ and CXCL17, or expressed and/or induced in tumor supporting cells [8,12,13];

- It is important to determine expression levels of all biomarker mRNAs also in control lymph nodes in order to establish back-ground levels [6,11];

- It is useful to express results as mRNA copies/normalization gene unit to be able to establish clinical cut-off levels by statistical method [7];

- Adapt the mRNA assay for use on formalin fixed tissue because it is easier to find lymph nodes in resected specimen after formalin fixation, analyses are directly comparable to examination of H\&E stained tissue and are compatible to clinical routine;

- Biomarker mRNAs can conveniently be analyzed in multiplex format which saves time and money and is most helpful for analysis of a large number of lymph nodes.

The criterium in biomarker mRNA selection that the marker identifying tumor cells or tumor supporting cells should not be expressed in immune cells can be difficult to fulfil, considering that immune cells include both $T$ and B lymphocytes, monocytes/macrophages/APCs, neutrophils and plasma cells, and activated and immature forms of these cells. Careful screening against a panel of these cell types is therefore necessary.

High levels of a biomarker mRNA may correlate with poor prognosis for the patient, KLK6 mRNA expression levels in lymph nodes of CRC patients being an excellent example [8]. However, the reverse may also occur. Thus, MUC2, the major mucin in colon epithelium, is a positive factor, supporting the notion that mucinous tumors are less aggressive compared with nonmucinous tumors [7]. A formula can be constructed where results from several biomarker mRNA analysis are included, both positive and negative factors, giving individual patients different results which may be correlated to patient survival.

I anticipate that assays based on a selected combination of biomarker mRNAs characterizing different properties of the tumor cells and their environment will be of great benefit to patients with CRC, reducing mortality and prolonging disease-free survival.

\section{Acknowledgments}

The author would like to thank ML Hammarström and G Lindmark for contributing to the final approval of the manuscript.

\section{Financial \& competing interests disclosure}

The financial support for the original research on which this editorial is based was received from the Swedish Cancer Society (PI: S Hammarström) and the Swedish Research Council-Natural Sciences and Engineering Sciences (PI: ML Hammarström). S Hammarström reported being a paid consultant to HiloProbe AB, Sweden (org. no. 559058-8918). The author has no other relevant affiliations or financial involvement with any organization or entity with a financial interest in or financial conflict with the subject matter or materials discussed in the manuscript apart from those disclosed.

No writing assistance was utilized in the production of this manuscript.

\section{References}

1. Böckelman C, Engelmann BE, Kaprio T, Hansen TF, Glimelius B. Risk of recurrence in patients with colon cancer stage II and III: a systematic review and meta-analysis of recent literature. Acta Oncol. 54(1), 5-16 (2015).

2. Jemal A, Siegel R, Ward E, Murray T, Xu J, Thun MJ. Cancer statistics, 2007. CA Cancer J. Clin. 57(1), 43-66 (2007).

3. Nicastri DG, Doucette JT, Godfrey TE, Hughes SJ. Is occult lymph node disease in colorectal cancer patients clinically significant? A review of the relevant literature. J. Mol. Diagn. 9(5), 563-571 (2007). 
4. Edge SB, Compton CC. The American joint committee on cancer: the 7th edition of the AJCC cancer staging manual and the future of TNM. Ann. Surg. Oncol. 17(6), 1471-1474 (2010).

5. Tsai HL, Huang CW, Yeh YS et al. Factors affecting number of lymph nodes harvested and the impact of examining a minimum of 12 lymph nodes in stage I-III colorectal cancer patients: a retrospective single institution cohort study of 1167 consecutive patients. $B M C$ Surgery 16(1), 17 (2016).

6. Ohlsson L, Hammarström ML, Israelsson A et al. Biomarker selection for detection of occult tumour cells in lymph nodes of colorectal cancer patients using real-time quantitative RT-PCR. Br. J. Cancer 95(2), 218-225 (2006).

7. Ohlsson L, Israelsson A, Öberg $\AA$ et al. Lymph node CEA and MUC2 mRNA as useful predictors of outcome in colorectal cancer. Int. J. Cancer 130(8), 1833-1843 (2012).

8. Ohlsson L, Lindmark G, Israelsson A et al. Lymph node tissue kallikrein-related peptidase 6 mRNA - a progression marker for colorectal cancer. Br. J. Cancer 107(1), 150-157 (2012).

9. Waldman SA, Hyslop T, Schulz S et al. Association of GUCY2C expression in lymph nodes with time to recurrence and disease-free survival in pN0 colorectal cancer. JAMA 301(7), 745-752 (2009).

10. Bas A, Forsberg G, Hammarström S, Hammarström ML. Utility of the housekeeping genes $18 S$ rRNA, $\beta$-actin and glyceraldehyde-3-phosphate-dehydrogenase for normalization in real-time quantitative reverse transcriptase-polymerase chain reaction analysis of gene expression in human T lymphocytes. Scand. J. Immunol. 59(6), 566-573 (2004).

11. Öberg ÅN, Lindmark GE, Israelsson AC, Hammarström SG, Hammarström ML. Detection of occult tumor cells in lymph nodes of colorectal cancer patients using real-time quantitative RT-PCR for CEA and CK20 mRNAs. Int. J. Cancer 111(1), 101-110 (2004).

12. Olsson L, Hammarström ML, Lindmark G, Hammarström S, Sitohy B. Ectopic expression of the chemokine CXCL17 in colon cancer cells. Br. J. Cancer 114(6), 697-703 (2016).

13. Rashad Y, Olsson L, Israelsson A et al. Lymph node CXCL17 messenger RNA: a new prognostic biomarker for colon cancer. Tumor Biol. 40(9), 1010428318799251 (2018). 
